\title{
Sublaminar fixation for traumatic lumbar fracture subluxation with lateral listhesis in a 2-year-old patient
}

\author{
Daniel S. Yanni, MD, ${ }^{1}$ Aurora S. Cruz, MD, MBA, ${ }^{2}$ Alexander Y. Halim, MD, ${ }^{3}$ Amandip S. Gill, MD, ${ }^{4}$ \\ Michael G. Muhonen, MD, ${ }^{5}$ Robert F. Heary, MD, ${ }^{6}$ and Ira M. Goldstein, MD ${ }^{6}$ \\ ${ }^{1}$ Disc Comfort, Inc., Newport Beach, California; ${ }^{2}$ Department of Neurosurgery, University of Louisville, Kentucky; ${ }^{3}$ Department \\ of Orthopaedic Surgery, University of California, Irvine, Orange, California; ${ }^{4}$ Heera Neurosurgical Associates, Inc., Northridge, \\ California; ${ }^{5}$ Department of Neurological Surgery, Children's Hospital of Orange County, Orange, California; and ${ }^{6}$ Department of \\ Neurological Surgery, Rutgers New Jersey Medical School, Newark, New Jersey
}

Pediatric spinal trauma can present a surgeon with difficult management decisions given the rarity of these cases, pediatric anatomy, and a growing spine. The need to stabilize a traumatically unstable pediatric spine can be an operative challenge given the lack of instrumentation available. The authors present a surgical technique and an illustrative case that may offer a novel, less disruptive method of stabilization. A 2-year-old girl presented after an assault with an L1-2 fracture subluxation with lateral listhesis and fractured jumped facets exhibited on CT scans. CT also showed intact growth plates at the vertebral body, pedicles, and posterior elements. MRI showed severe ligamentous injury, conus medullaris compression, and an epidural hematoma. Neurologically, the patient moved both lower extremities asymmetrically. Given the severity of the deformity and neurological examination and disruption of the stabilizing structures, the authors made the decision to surgically decompress the L-1 and L-2 segments with bilateral laminotomies, evacuate the epidural hematoma, and reduce the deformity with sublaminar stabilization using braided polyester cables bilaterally, thus preserving the growth plates. They also performed a posterolateral onlay fusion at L-1 and L-2 using autograft and allograft placed due to the facet disruption. At the 42-month follow-up, imaging showed fusion of L-1 and L-2 with good alignment, and the hardware was subsequently explanted. The patient was neurologically symmetric in strength, ambulating, and had preserved alignment. Her bones and spinal canal continued to grow in relation to the other levels. https://thejns.org/doi/abs/10.3171/2018.1.PEDS1648

KEYWORDS pediatric; lumbar spine; trauma; stabilization; fusion; sublaminar cables; polyester bands; sublaminar wires; fracture; listhesis; Universal Clamp

$\mathrm{P}$ EDIATRIC spinal trauma can present a surgeon with difficult management decisions. Pediatric spinal injuries account for only $2 \%-3 \%$ of all spinal column injuries. ${ }^{5,8,10,19}$ In particular, traumatic lumbar spine instability is exceedingly rare. ${ }^{8}$ Most often, lumbar instability is the result of high-energy trauma, and neurological recovery is strongly indicated by the extent of soft-tissue damage. ${ }^{17,19}$

Invasive surgical procedures may disrupt growing structures at different times during development. Thus, it is important to review the embryology and development of the spine for pediatric spinal surgery. Vertebral ossification usually begins during the 7 th week of age and can extend to the 25 th year. ${ }^{11}$ Three primary centers of ossifi- cation in the vertebrae form by the 8th week of fetal development. ${ }^{11}$ There is one center of ossification on each half of the neural arch and one in the centrum. Of the 3 bony parts of the vertebrae, the vertebral arches are the first to fuse between the ages of 3 and 5 years. The vertebral arch also articulates with the centrum at the cartilaginous growth plate, referred to as the neurocentral synchondrosis. ${ }^{11,15}$ While this joint allows for vertebral growth during spinal cord growth, it ossifies and disappears between the ages of 3 and 6 years. In particular, the lumbar region of the spine is the first section to undergo vertebral fusion. Thus, identification and preservation of the neurocentral synchondrosis may be an important factor in planning for pediatric spinal surgeries. 

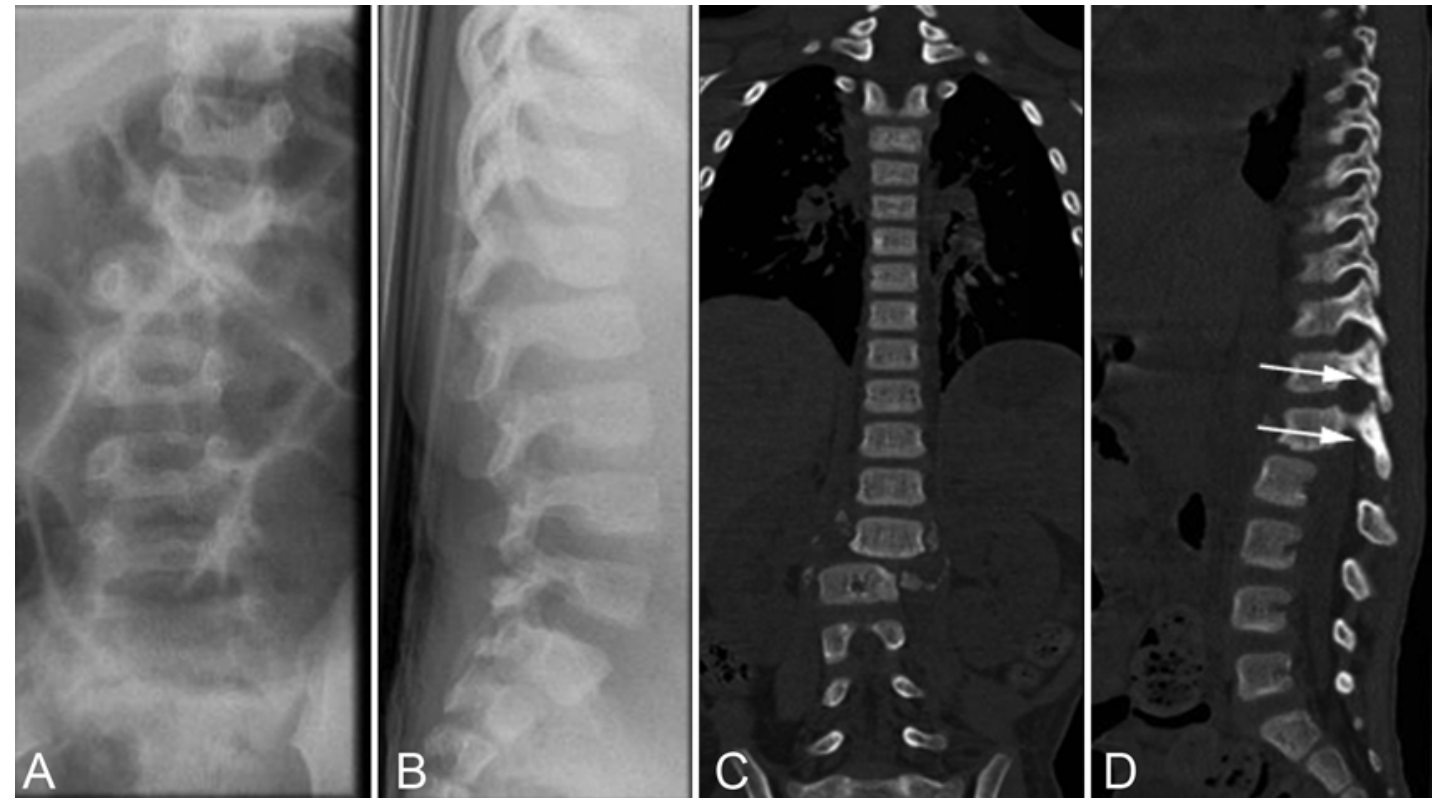

FIG. 1. Initial anteroposterior (A) and lateral (B) radiographs and coronal (C) and sagittal (D) CT scans showing an L-1 and L-2 fracture subluxation, lateral listhesis, fractured/jumped facets, and intact growth plates (arrows) at the vertebral body, pedicles, and posterior elements.

Pediatric lumbar spine injuries involving fracture subluxation with lateral listhesis have not been well documented. Four cases on traumatic lumbar spine dislocations surgically addressed from a posterior approach have been reported in literature, with patient ages ranging from 6 to 17 years. ${ }^{4,10,17,19}$ Only one case report by Yazici et al. described the dislocation of L-1 and L-2 segments in a 6-year-old girl after traumatic injury; in this case, the authors employed a modified Luque frame of braided wires extending from T-12 to L-3 with good surgical outcome. ${ }^{19}$

Because of its rare occurrence, there is a lack of literature and guidelines for the treatment of pediatric lumbar injury. This is attributable to the pediatric bony spine durability and its surrounding immature musculature, liga-
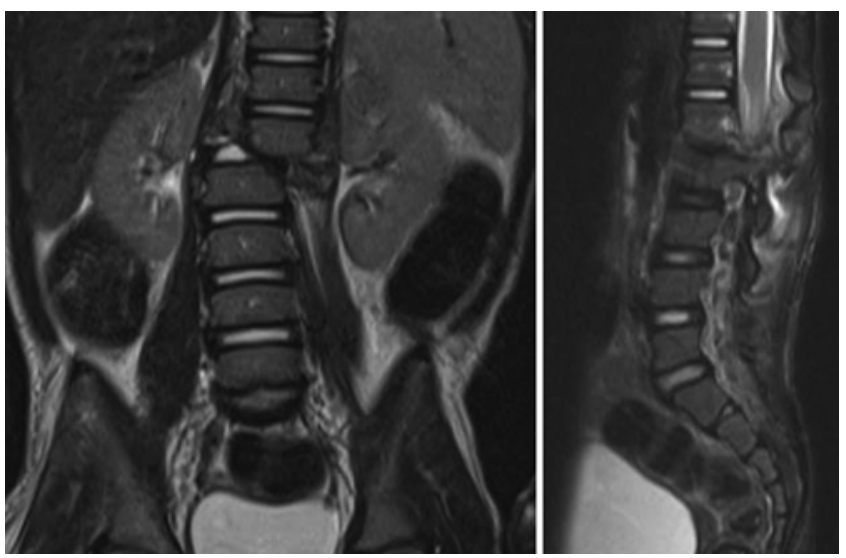

FIG. 2. Coronal (left) and sagittal (right) T2-weighted MR images showing severe ligamentous injury, conus medullaris compression, and epidural hematoma at the L-1 and L-2 levels. ments, and growth plates. ${ }^{9}$ The need to stabilize a traumatically unstable spine in a pediatric patient presents an operative challenge. Protecting neural tissue, restoring alignment, and preserving spine growth plates are critical considerations in the treatment plan of this patient population. We present a surgical technique and an illustrative case in the youngest patient reported in the literature to offer a novel and less disruptive method of stabilization.

\section{Case Report}

A 2-year-old girl presented to the emergency department after an assault awake and alert but in significant discomfort. Neurologically, the patient had preserved sensation to pain and reflexes bilaterally. She was also able to move both lower extremities but asymmetrically, with the right lower extremity more limited in motion. Due to the patient's age and multiple traumatic injuries including a right femur fracture, we were unable to complete full neurological assessment other than to determine that her spinal cord injury was incomplete and she had some motor dysfunction. Bowel and bladder dysfunction were unable to be fully assessed due to patient's age.

Radiological examination revealed an L-1 and L-2 fracture subluxation, lateral listhesis, and jumped fractured facets on CT scan and x-ray radiography (Fig. 1). CT scan also showed intact growth plates at the vertebral body, pedicles, and posterior elements (Fig. 1). MRI showed severe ligamentous injury, conus medullaris compression, $1.5 \mathrm{~cm}$ of lateral listhesis, and an epidural hematoma (Fig. 2). Imaging was also significant for a right femur fracture. Given the imaging and clinical findings, we made the decision to urgently surgically decompress the patient's L-1 and L-2 segments posteriorly with bilateral laminotomies at L-1 and L-2 and foraminotomies bilater- 


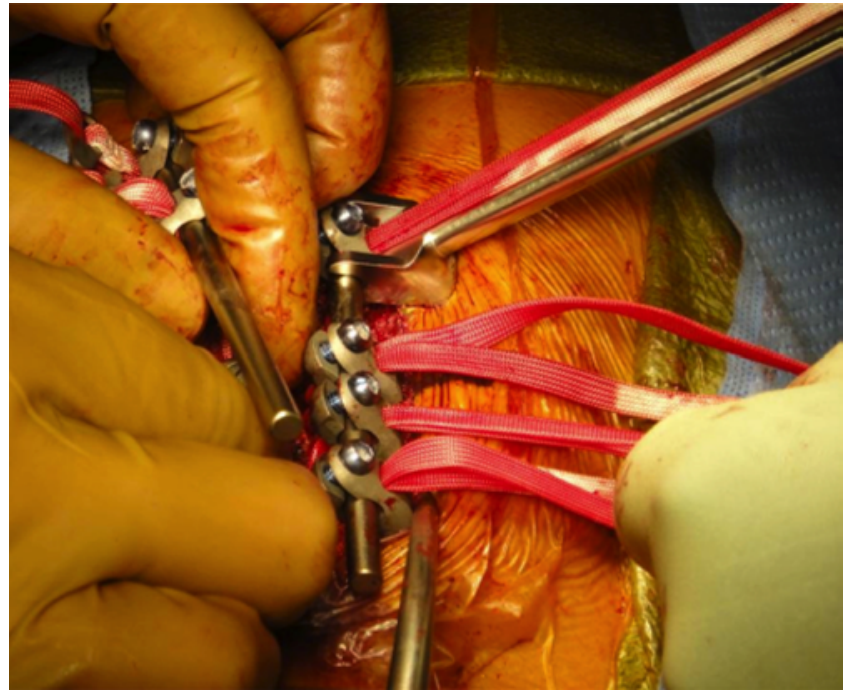

FIG. 3. Intraoperative photograph showing placement of the Zimmer Universal Clamp braided polyester sublaminar cables connected to a $5.5-\mathrm{mm}$ rod. Figure is available in color online only.

ally at L1-2, place braided polyester sublaminar cables at T-12 to L-3 via smaller laminotomies, reduce the subluxation and lateral listhesis, fuse L-1 and L-2 with autograft and allograft, and evacuate the epidural hematoma to decompress the conus medullaris.

After a midline posterior thoracolumbar approach, we placed 8 Universal Clamp (Zimmer) cables in the sublaminar spaces bilaterally. To accomplish this, a matchstick drill bit was used to make a laminotomy at each level, and an up-biting curette was then used to dissect free the ligamentum flavum. The metal passer at the leading edge of the Universal Clamp band was gently bent to allow for passage from inferior to superior at the T-12, L-1, L-2, and L-3 levels (Fig. 3). This was performed in order to reduce the lateral listhesis, preserve the patient's growth plates, and stabilize the segmental instability at L1-2. After implanting the 8 Universal Clamp cables, 5.5-mm titanium rods were measured and cut. We then attached each Universal Clamp to the rods at their respective locations. Optimal tension was then applied to each band using the tensioning tool (Fig. 4). The sequence of tensioning was as follows: left L-2, left L-3, right L-1, right T-12, left L-1, left $\mathrm{T}-12$, right L-2, and then right L-3. Excellent reduction and stabilization was achieved (Fig. 5). We then performed a posterolateral onlay fusion at L-1 and L-2 using autograft and demineralized bone matrix allograft due to the facet fractures and disruption (Figs. 5 and 6A). Neuromonitoring was not used during this procedure, as it was not available at the time of surgery. Because of the prominence of the hardware, we mobilized the paraspinal muscles to allow for adequate cushioning and closure of the skin. The patient had an uncomplicated postoperative recovery. She was placed in a brace and transferred to a pediatric rehabilitation facility and was able to move her bilateral lower extremities symmetrically.

At the 9-month follow-up, the patient's stabilization appeared successful, with fusion of the L-1 and L-2 facet complex. The allograft and autograft were both well in-

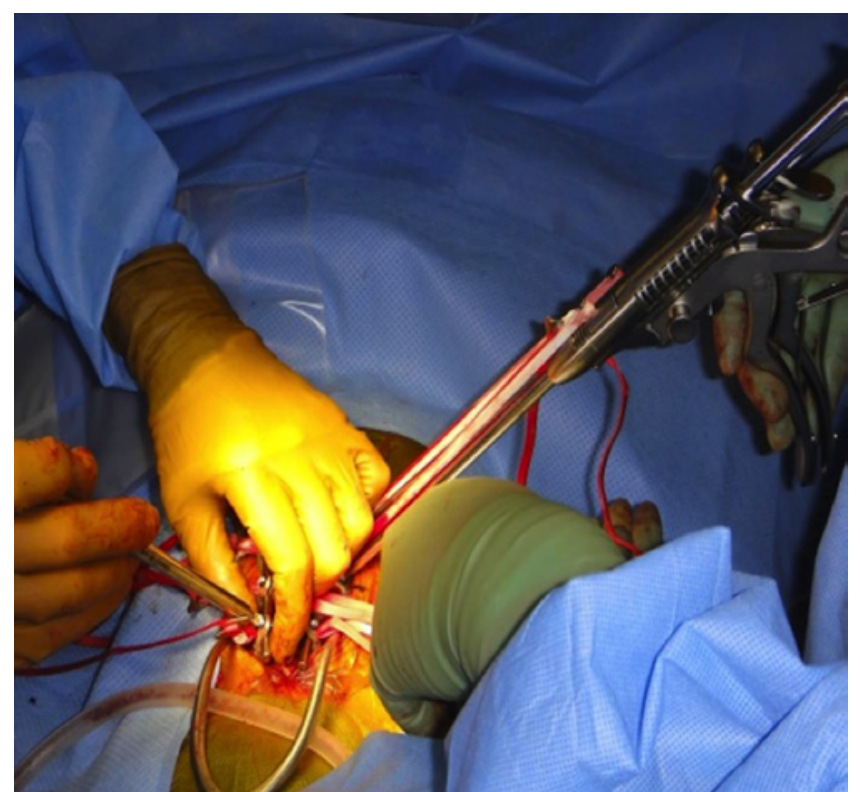

FIG. 4. Intraoperative photograph showing sequential tensioning of cables for reduction of the deformity. Figure is available in color online only.

corporated in the posterolateral gutters, with significant fracture reduction (Fig. 6B). Clinically, the patient's lowerextremity strength had become symmetric, and her bladder control had improved, according to her foster parents. At 16 months after the index procedure, the hardware was explanted, and excellent fusion of L1-2 and preservation of the adjacent facets was seen (Fig. 7). The patient was neurologically intact and symmetric in strength, sensation, bowel and bladder function, and ambulation. Her bones and spinal canal continued to grow in relation to the other levels with intact growth plates at the vertebral bodies, pedicles, and posterior elements (Figs. 8 and 9). The patient exhibited preserved alignment and thoracic and lumbar curves on radiographic imaging (Fig. 9). At

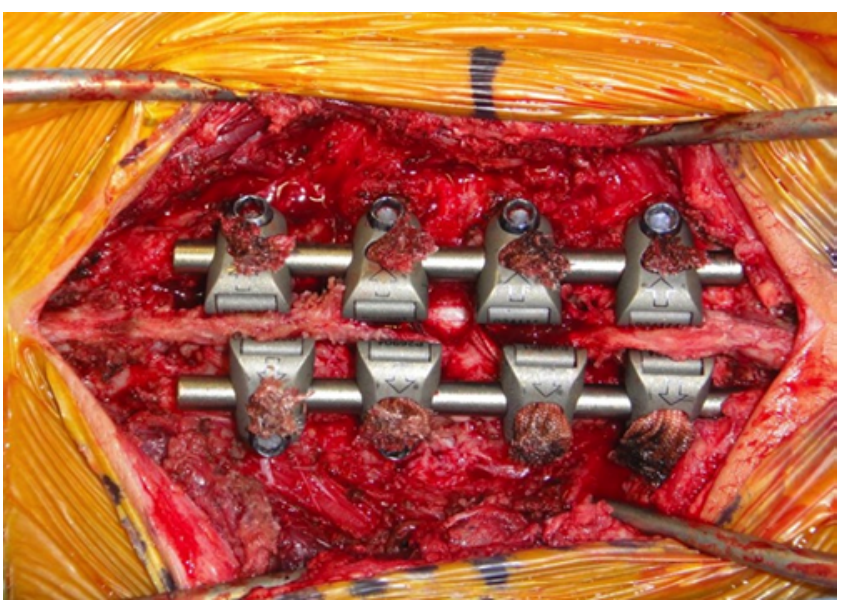

FIG. 5. Final intraoperative photograph showing the cable-rod construct for L1-2 decompression and fusion. Figure is available in color online only. 


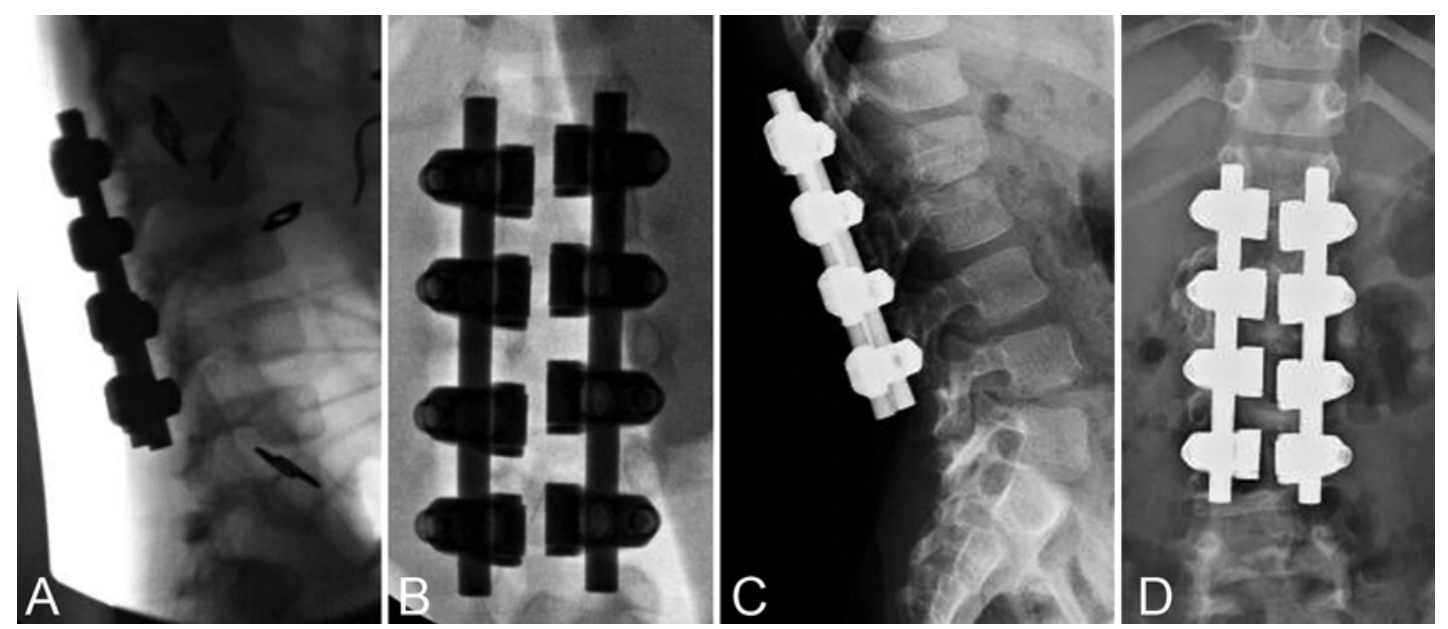

FIG. 6. Postoperative lateral (A) and anteroposterior (B) radiographs of the T12-L3 region obtained immediately postoperatively, and lateral (C) and anteroposterior (D) lumbar radiographs obtained at the 9-month follow-up.

42 months after the index procedure, the patient remained neurologically intact without pain or limitation in activities and advanced to the 50th percentile in height for age from

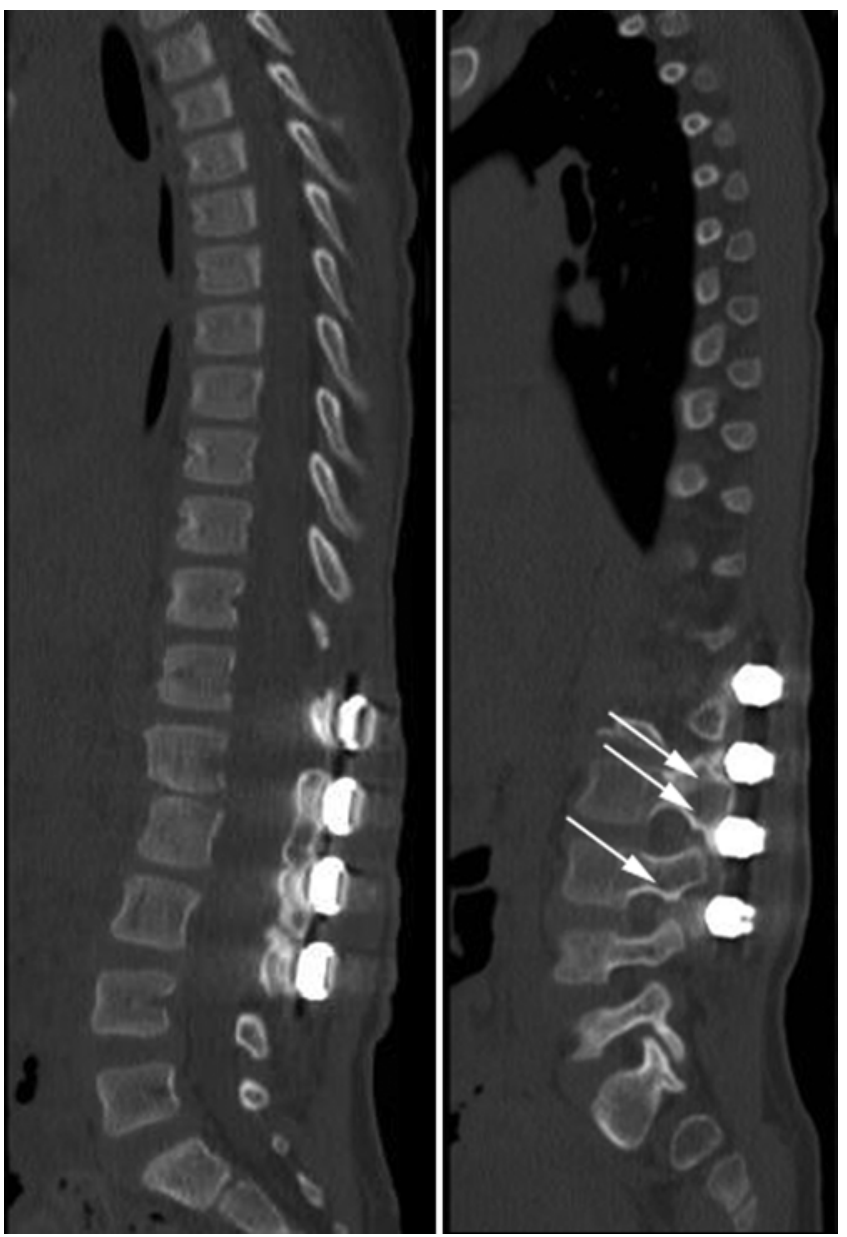

FIG. 7. Sagittal CT scans obtained 1 year postoperatively but before hardware removal, showing sublaminar stabilization with Universal Clamp braided polyester cables bilaterally at the T-12, L-1, L-2, and L-3 levels with fusion at L1-2 and preservation of the growth plates (arrows). the 10th percentile at the time of injury, showing age appropriate growth despite previous short stature and singlelevel lumbar fusion. ${ }^{6}$ By using braided cables and avoiding disruption of the neurocentral synchondroses with pedicle screws, this procedure allowed the child's spinal canal to continue to grow normally, as demonstrated on postoperative MR images (Figs. 8 and 10).

\section{Discussion}

Spinal injuries are rare in young children because this patient population has more elastic ligaments and vertebral columns with greater cartilaginous content than
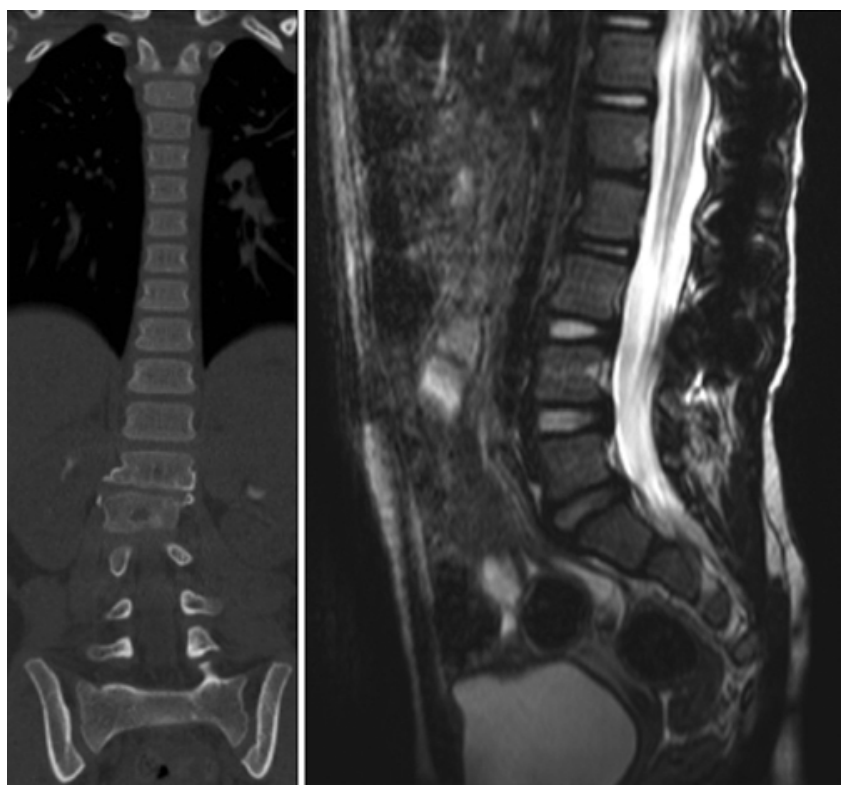

FIG. 8. Coronal CT scan (left) and sagittal T2-weighted MR image (right) obtained 1 year postoperatively but before hardware removal, showing continued bone and spinal canal growth in relation to the other levels with intact growth plates at the vertebral bodies, pedicles, and posterior elements. 

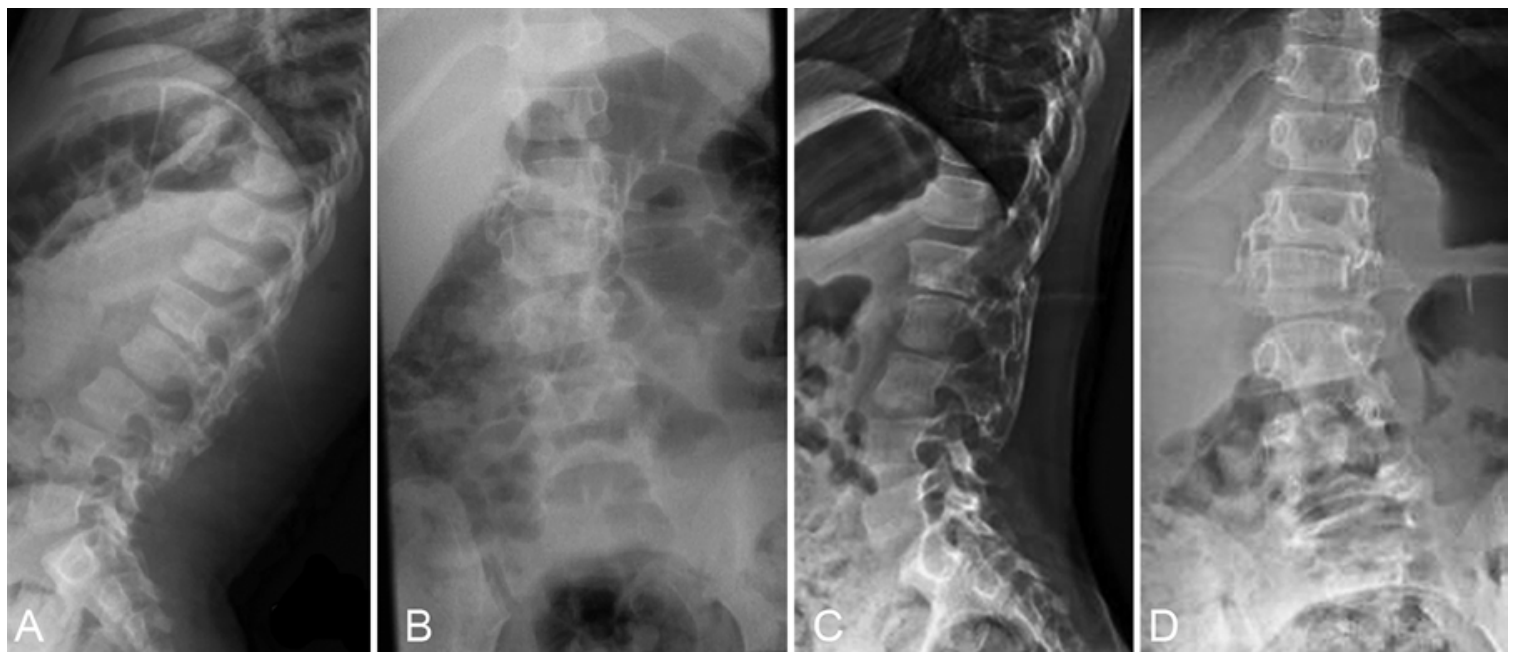

FIG. 9. Standing lateral (A) and anteroposterior (B) radiographs obtained immediately after hardware removal. Standing lateral (C) and anteroposterior (D) radiographs obtained 42 months after the index procedure, demonstrating preserved alignment with thoracic and lumbar curvature.

adults. ${ }^{4,7,8,19}$ Furthermore, pediatric lumbar fracture-dislocations are exceedingly rare and are almost always caused by high-energy trauma. Neurological and functional recovery is dependent on the extent of tissue damage, which can be difficult to assess. ${ }^{5,19}$

In order to demonstrate the importance of maintaining spinal alignment in the pediatric population, Cil et al. published a study demonstrating that the pediatric sagittal curvature in the thoracolumbar spine is significantly more dynamic than that in the adult spine and will change with development. ${ }^{3}$ This group strongly recommended exercising special care when planning to implant instrumentation in a pediatric spine in order to avoid sagittal malalignment. In the case we present, the patient maintained excellent alignment after hardware removal and at 42 months after the index procedure, as shown on the standing anteroposterior and lateral radiographs (Fig. 9).

In the past, placement of pedicle screw fixation constructs was the most commonly used procedure to correct pediatric spinal deformities. . $^{2,15,15,19}$ Sairyo et al. studied the efficacy of the pedicle screw instrumentation on 10 immature thoracic and lumbar calf vertebrae and found that the immature lumbar vertebrae are uniquely weak and susceptible to fracture when using pedicle screws due to the neurocentral synchondrosis. ${ }^{15}$ The authors further warned that surgeons must be very careful when deciding to use pedicle screws in very young children because of their potential to fracture and disrupt lumbar growth plates. As a less invasive and disruptive alternative, our case offers a unique treatment option by using braided polyester sublaminar cables to stabilize the patient's thoracolumbar segment, providing an effective temporary internal brace.

Previous studies have raised concern for the safety of sublaminar wire-only constructs when compared to pedicle screw-only constructs, finding that the rates of new neurological deficits were $1.7 \%$ and $0.7 \%$, respectively. ${ }^{14}$ Although the technique is similar, sublaminar use of pliable braided polyester cables has been shown to be safe, and the risks are not equivalent to stainless steel wiring. Braided polyester was chosen in this case because of its additional surface area and elasticity as well as the fear of stainless steel sublaminar wires shearing through the lamina due to the degree of tension needed to reduce the deformity. In assessing the efficacy and safety of braided polyester sublaminar cables, Mazda et al. compared the Universal Clamp to laminar hooks, pedicle screws, and sublaminar wires in adolescents with idiopathic scoliosis. ${ }^{9}$ They found that the Universal Clamp technique reduced operative time, radiation exposure, and blood loss and had superior correction in the sagittal plane. The biocompatibility of polyester bands has been well documented and used in spinal implants in Europe for over 20 years. ${ }^{15,18}$ We opted for this approach because the polyester bands
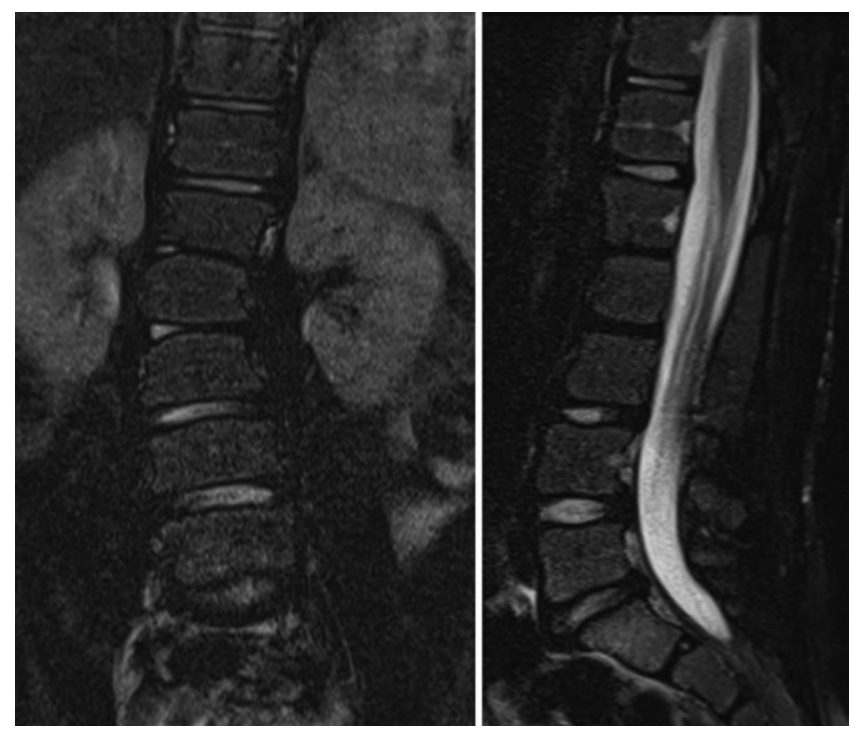

FIG. 10. Coronal (left) and sagittal (right) T2-weighted MR images obtained 42 months postoperatively, showing intact growth plates at the vertebral bodies, pedicles, and posterior elements. 
provide a greater surface area to distribute tension over the lamina to allow for reduction of the deformity while allowing the greatest probability for growth plate preservation. ${ }^{3,9}$ This is a critical consideration due to the fact that the lumbar neurocentral synchondrosis cartilaginous growth plates ossify between the ages of 3 and 5 years, leaving our patient uniquely susceptible to impaired growth and the possibility of an iatrogenic developmental stenosis. ${ }^{11}$ Additionally, we performed an L-1 and L-2 posterolateral onlay fusion in order to provide long-term stability and compensate for our patient's severe ligamentous injury and facet fractures. Long-construct instrumentation was chosen to allow for the forces necessary to reduce the fracture subluxation deformity.

Viswanathan et al. presented 5 cases of pediatric posterior instrumentation with hybrid hook-screw-sublaminar polyester band constructs; their conclusion is consistent with our findings that sublaminar polyester bands are ideal for the smaller, skeletally immature pediatric spine due to decreased contact stresses at the bone-band interface, while also providing the immediate biomechanical rigidity of pedicle screws and hooks. ${ }^{18}$ Strickland et al. described the use of subtransverse process polyester bands in posterior instrumented fusion in 4 pediatric patients for nontraumatic deformity correction and found low risk of neurological injury and adequate biomechanical stability. ${ }^{16}$ Viswanathan et al. also identified the concern regarding the proper amount of tension placed on the band, as excess tension may lead to laminar fracture. ${ }^{18}$ Further studies are needed to help guide the proper tension per age group, although careful attention to this detail during surgery is crucial. Polirsztok et al. published a series of 378 spine deformity correction procedures in children and adolescents in which they used a hybrid posterior approach including lumbar screws, hooks, and thoracic sublaminar bands. ${ }^{13}$ This group found that sublaminar bands offer safety comparable to that of other spinal implants in the pediatric population. Despite the young age at presentation, authors including Verhelst et al. and Aihara et al. agree that pediatric lumbar fractures with dissociation should be treated with posterior stabilization. ${ }^{1,17}$ Only a single case report by Yazici et al. has addressed traumatic dislocation of the L-1 and L-2 levels in a 6-year-old child, using a modified Luque frame to correct the dislocation and sublaminar wiring with allograft bone fixation, with good fusion results at follow-up, similar to our case. ${ }^{19}$ In the case presented by Verhelst et al., a traumatic retrolisthesis of the lumbosacral junction with transection of the neural elements in a 6-year-old male was treated by performing laminectomies from L-4 to S-1 and placing pedicle screws from L-3 to S-2 (excluding L-5) for lumbosacral fixation. At the 1-year follow-up, good fracture reduction and posterior fusion were achieved without recovery of neural function below L-3. ${ }^{17}$ In both cases presented, the authors used different instrumentation options available to reduce deformity and stabilize the pediatric spine. The case we present posed a unique challenge in comparison to the cases reported by Yazici and Verhelst and their colleagues with respect to this patient's age and the presence of open neurocentral synchondroses.

In the case we present, we used a construct similar to that used by Yazici et al. to successfully preserve the neurocentral synchondroses while achieving reduction of deformity with stabilization; however, the braided polyester sublaminar cables allowed for a more straightforward explantation. For the explantation procedure, we cut the polyester cables with a scalpel and removed the rods. The intent in this case was to confine the fusion to the L1-2 segment; on postoperative imaging there is definitive evidence of bony fusion at L1-2. There is also some evidence that T12-L1 and L2-3 have fused based on the postoperative radiography findings (Fig. 9), although CT and MRI both demonstrate open and unfused facet joints and disc spaces (Figs. 7, 8, and 10).

The hardware was explanted 16 months after the index procedure; at 42 months postoperatively, our patient exhibited full neurological strength symmetrically, full ambulation, bladder continence, and preserved alignment. Her bones and spinal canal continued to grow in relation to the other levels. These results demonstrate and support the efficacy of using Universal Clamp braided polyester cables in a pediatric setting with the goal of preserving growth plates, correcting the deformity, and allowing for early rehabilitation and ambulation.

Traumatic fracture and subluxation with lateral listhesis in a pediatric patient is exceedingly rare. We present this case to address the operative challenge of stabilizing the pediatric patient's lumbar region while preserving neurological function and open growth plates at the vertebral body, pedicles, and posterior elements. This case describes the youngest patient presented in the literature with traumatic fracture subluxation with lateral listhesis. Followup at 4 years after index procedure revealed an excellent clinical and radiographic outcome. Thus, sublaminar fixation is a safe and effective treatment option in addressing these rare traumatic spinal injuries. More follow-up studies should be conducted to assess the outcomes of posterior spinal stabilization with braided polyester cables in pediatric spinal trauma as well as studies assessing longterm efficacy.

\section{References}

1. Aihara T, Takahashi K, Yamagata M, Moriya H: Fracturedislocation of the fifth lumbar vertebra. A new classification. J Bone Joint Surg Br 80:840-845, 1998

2. Boos N, Marchesi D, Zuber K, Aebi M: Treatment of severe spondylolisthesis by reduction and pedicular fixation. A 4-6year follow-up study. Spine (Phila Pa 1976) 18:1655-1661, 1993

3. Cil A, Yazici M, Uzumcugil A, Kandemir U, Alanay A, Alanay Y, et al: The evolution of sagittal segmental alignment of the spine during childhood. Spine (Phila Pa 1976) 30:93-100, 2005

4. Cohn SL, Keppler L, Akbarnia BA: Traumatic retrolisthesis of the lumbosacral junction. A case report. Spine (Phila Pa 1976) 14:132-134, 1989

5. Crawford AH: Operative treatment of spine fractures in children. Orthop Clin North Am 21:325-339, 1990

6. De Onis M (ed): WHO Child Growth Standards. Geneva: WHO, 2006 (http://www.who.int/childgrowth/standards/ Technical_report.pdf) [Accessed March 6, 2018]

7. Dimeglio A: Growth in pediatric orthopaedics, in Morrissy RT, Weinstein SL (eds): Lovell and Winter's Paediatric Orthopaedics. Philadelphia: Lippincott Williams \& Wilkins, 2006, pp 35-65 
8. Dogan S, Safavi-Abbasi S, Theodore N, Chang SW, Horn EM, Mariwalla NR, et al: Thoracolumbar and sacral spinal injuries in children and adolescents: a review of 89 cases. J Neurosurg 106 (6 Suppl):426-433, 2007

9. Mazda K, Ilharreborde B, Even J, Lefevre Y, Fitoussi F, Penneçot GF: Efficacy and safety of posteromedial translation for correction of thoracic curves in adolescent idiopathic scoliosis using a new connection to the spine: the Universal Clamp. Eur Spine J 18:158-169, 2009

10. Meneghini RM, DeWald CJ: Traumatic posterior spondyloptosis at the lumbosacral junction. A case report. J Bone Joint Surg Am 85-A:346-350, 2003

11. Moore K, Persaud T, Torchia M: The Developing Human: Clinically Oriented Embryology, ed 9. Philadelphia: Elsevier Saunders, 2013

12. Ohlin A, Karlsson M, Düppe H, Hasserius R, RedlundJohnell I: Complications after transpedicular stabilization of the spine. A survivorship analysis of 163 cases. Spine (Phila Pa 1976) 19:2774-2779, 1994

13. Polirsztok E, Gavaret M, Gsell T, Suprano I, Choufani E, Bollini G, et al: Sublaminar bands: are they safe? Eur Spine J 24:1441-1449, 2015

14. Reames DL, Smith JS, Fu KM, Polly DW Jr, Ames CP, Berven SH, et al: Complications in the surgical treatment of 19,360 cases of pediatric scoliosis: a review of the Scoliosis Research Society Morbidity and Mortality database. Spine (Phila Pa 1976) 36:1484-1491, 2011

15. Sairyo K, Scifert J, Goel VK, Grosland N, Grobler LJ: Neurocentral synchondrosis fracture in immature spines associated with pedicle screw type fixation devices. J Spinal Disord 11:142-145, 1998

16. Strickland BA, Sayama C, Briceño V, Lam SK, Luerssen TG, Jea A: Use of subtransverse process polyester bands in pediatric spine surgery: a case series of 4 patients with a minimum of 12 months' follow-up. J Neurosurg Pediatr 17:208-214, 2016
17. Verhelst L, Ackerman P, Van Meirhaeghe J: Traumatic posterior lumbosacral spondyloptosis in a six-year-old: a case report and review of the literature. Spine (Phila Pa 1976) 34:E629-E634, 2009

18. Viswanathan A, Johnson KK, Whitehead WE, Curry DJ, Luerssen TG, Jea A: Hybrid spinal constructs using sublaminar polyester bands in posterior instrumented fusions in children: a series of 5 cases. Neurosurgery 66:862-867, 2010

19. Yazici M, Alanay A, Aksoy MC, Acaroglu E, Surat A: Traumatic L1-L2 dislocation without fracture in a 6-year-old girl. Incomplete neurologic deficit and total recovery. Spine (Phila Pa 1976) 24:1483-1486, 1999

\section{Disclosures}

Dr. Yanni: consultant for Zimmer Biomet, DePuy Spine, and NuVasive. Dr. Goldstein: consultant for Zimmer Spine, Stryker Spine, Alphatec Spine, and Biomet; honorarium from Biomet; and travel expenses from Globus.

\section{Author Contributions}

Conception and design: all authors. Acquisition of data: Yanni, Halim, Gill, Muhonen. Analysis and interpretation of data: Yanni, Halim, Gill, Muhonen. Drafting the article: Yanni, Cruz, Halim. Critically revising the article: Yanni, Cruz, Gill, Muhonen, Heary, Goldstein. Reviewed submitted version of manuscript: all authors. Approved the final version of the manuscript on behalf of all authors: Yanni. Study supervision: Cruz, Gill, Heart, Goldstein.

\section{Correspondence}

Daniel S. Yanni: Disc Comfort, Inc., Newport Beach, CA. dyannimd@hotmail.com. 